\title{
Comparative transcriptome analysis between metastatic and non-metastatic gastric cancer reveals potential biomarkers
}

\author{
DAN FENG ${ }^{1}$, XIAOFEI YE ${ }^{2}$, ZHENXIN ZHU $^{3}$, ZIRAN WEI $^{3}$, QINGPING CAI $^{3}$ and YAJIE WANG ${ }^{1}$ \\ ${ }^{1}$ Department of Oncology, Changhai Hospital; ${ }^{2}$ Department of Health Statistics, Second Military Medical University, \\ Shanghai 200433; ${ }^{3}$ Department of Gastrointestine Surgery, Shanghai Changzheng Hospital, Shanghai 200003, P.R. China
}

Received December 20, 2013; Accepted July 4, 2014

DOI: $10.3892 / \mathrm{mmr} .2014 .2709$

\begin{abstract}
The transcriptome of metastatic gastric cancer (GC) was compared to that of non-metastatic GC to identify metastasis-related biomarkers. The gene expression dataset GSE21328, comprising 2 metastatic GC samples and 2 non-metastatic GC samples, was downloaded from the Gene Expression Omnibus database. Differential expression analysis was performed with the package limma of Bioconductor to identify differentially expressed genes (DEGs). Gene Ontology (GO) enrichment analysis was performed to identify significantly altered biological functions. In addition, the transcriptional regulatory and protein-protein interaction networks were constructed with information from the UCSC genome browser and STRING database, respectively, followed by functional enrichment analysis of all of the genes in these two networks. A total of 584 DEGs were identified, of which 175 were upregulated and 409 downregulated. Clustering analysis confirmed that these genes can distinguish metastatic from non-metastatic GC. Upregulated genes were enriched for the xenobiotic metabolic process, while downregulated genes were enriched for immune response and related pathways. Among the 584 DEGs, six genes (DAND5, EGR2, FOXD1, LMO2, PRRX2 and STAT1) were shown to encode transcription factors, which were used to establish the transcriptional regulatory network with 169 target genes, forming 175 nodes. The proteins of this network were significantly enriched for the process of negative regulation of cell differentiation. In conclusion, this study identified a range of DEGs in meta-
\end{abstract}

Correspondence to: Dr Yajie Wang, Department of Oncology, Changhai Hospital, 168 Changhai Road, Shanghai 200433, P.R. China

E-mail: yajiewangyjw@hotmail.com

Dr Qingping Cai, Department of Gastrointestine Surgery, Shanghai Changzheng Hospital, 415 Fengyang Road, Shanghai 200003, P.R. China

E-mail: qingpingcai@hotmail.com

Key words: metastatic gastric cancer, biomarker, gene expression data, differentially expressed genes, functional enrichment analysis, transcriptional regulatory network static GC, which may enhance our current knowledge on this disease. Among these genes, STAT1 and EGR2 may constitute potential biomarkers of GC metastasis.

\section{Introduction}

Gastric cancer (GC) is the fourth most common cancer worldwide. It causes $\sim 800,000$ deaths/year, which renders it the second leading cause of cancer-related mortality after lung cancer (1). Metastasis occurs in $80-90 \%$ of individuals with GC, with a six-month survival rate of $65 \%$ for patients diagnosed at the early stages, and $<15 \%$ for those diagnosed at late stages. Therefore, metastasis-related biomarkers are necessary to allow early diagnosis and development of targeted therapies.

A number of studies have demonstrated that diverse signaling pathways are involved in the metastasis of GC. Yonemura et al (2) demonstrated that cancer cells producing VEGF-C induces proliferation and dilation of lymphatic vessels, resulting in the invasion of cancer cells into the lymphatic vessel and lymph node metastasis. Shimizu et al (3) further showed that inhibition of VEGFR-3 signaling inhibits lymph node metastasis of GC cells. Xu et al (4) found that expression of receptors for advanced glycation end-products is closely associated with the invasive and metastatic activity of GC cells. The tumor microenvironment also appears to considerably affect metastasis. Upregulation of manganese superoxide dismutase was found in metastatic GC, which may be associated with the reactive oxygen status of the gastric tumor microenvironment (5). The epithelial cell adhesion molecule E-cadherin also plays a role in tumorigenesis and metastasis of GC cells (6). Given the poor outcome of patients with metastatic GC, additional research is necessary to unveil the molecular mechanisms underlying metastasis, and thus provide useful biomarkers for early diagnosis and treatment.

Microarray technology is an appropriate tool to investigate the global gene expression changes in cancers such as GC. Hippo et al (7) adopted this technology to study the global gene expression profiles in GC. Kang et al (8) identified differentially expressed genes (DEGs) in drug-resistant GC cells. Yamashita et al (9) carried out a genome-wide screening for methylation-silenced genes using 5-aza-2'-deoxycytidine treatment and oligonucleotide microarrays. 
By now, massive microarray data have been acquired. We considered that a comprehensive exploration of existing datasets with bioinformatic tools may provide novel insights into GC metastasis. Therefore, in the present study, a comparative analysis between metastatic and non-metastatic GC gene expression data was performed to identify DEGs, which may allow to reveal the molecular mechanisms underlying metastasis and provide potential biomarkers for the early diagnosis of this disease.

\section{Materials and methods}

Microarray data and pretreatment. The gene expression dataset GSE21328 was downloaded from the Gene Expression Omnibus database (10), and comprised 2 metastatic stomach cancer samples and 2 non-metastatic stomach cancer samples (11). The platform in which the data were acquired was the Agilent-014850 Whole Human Genome 4x44K G4112F microarray (feature number version).

Raw data were $\log _{2}$ transformed. Probes were mapped to genes according to the annotation file. The average expression level for each gene was calculated from the expression data for all probes corresponding to the same gene. Normalization of the data was performed with the Affy package of Bioconductor (12).

Identification of DEGs. The Bioconductor package limma (13) was chosen for differential expression analysis. As cut-offs, we used the log (fold-change) $>1.5$ and p-value $<0.05$. Hierarchical clustering of the DEGs was performed on median-centered, $\log _{2}$-transformed data using the Cluster software (14), and was subsequently visualized using Treeview software (15).

Functional enrichment analysis. Gene Ontology (GO) enrichment analysis was performed for upregulated and downregulated genes with DAVID (16). Terms with false discovery rate $(\mathrm{FDR})<0.05$ were retained, and then compared between the two groups.

Transcriptional regulatory network analysis. A transcriptional regulatory network was constructed for DEGs with information from the UCSC genome browser (http://genome.ucsc. edu) (17). GO and Kyoto Encyclopedia of Genes and Genomes (KEGG; 18) enrichment analysis were performed for the nodes in the network in order to identify proteins and pathways that were significantly enriched in metastasis. FDR $<0.05$ was set as the cut-off.

Construction of a protein-protein interaction (PPI) network. A PPI network was constructed for DEGs using information from the STRING database (http://string-db.org/). The interactions with score $>0.7$ were retrieved from the database and then visualized using the Cytoscape software platform (19). The proteins in the network were defined as the nodes and the degree of a node corresponds to the number of interactions of a protein. Functional enrichment analysis was applied on hub nodes with degree of $>5$. The Clustering with Overlapping Neighborhood Expansion (ClusterONE) plugin from Cytoscape (20) was used to extract functional modules from the entire network. Number of node $\geq 10$ and p-value $<0.05$ were set as the cut-offs.

\section{Results}

DEGs. The microarray dataset GSE21328 included 12,116 gene expression values. Normalization of these data was satisfactory (Fig. 1). A total of 584 DEGs were then identified for metastatic stomach cancer, of which 175 were upregulated and 409 were downregulated. The clustering analysis result is shown in Fig. 2, indicating these genes may be used to distinguish between metastatic and non-metastatic GC.

Functional enrichment analysis. Upregulated genes were enriched for the GO term 'xenobiotic metabolic process'. Downregulated genes were enriched for 'immune response', 'response to virus', 'antigen processing and presentation', and 'cell morphogenesis involved in differentiation'. In addition, a considerable portion of DEGs was predicted to locate at the extracellular space (Table I).

Transcriptional regulatory network. A total of 215 transcription factors (TFs) and 21,4607 TF-target gene interactions are included in the UCSC database. Out of the 584 DEGs, 6 downregulated genes were found to encode TFs. These TFs were: DAN domain family member 5 (DAND5), which is a BMP antagonist, early growth response 2 (EGR2), forkhead box D1 (FOXD1), LIM domain only 2 (LMO2), paired mesoderm homeobox protein 2 (PRRX2) and signal transducer and activator of transcription 1 (STAT1). No upregulated TFs were detected. A transcriptional regulatory network was constructed on the 6 TFs, and their 169 target genes (Fig. 3), forming 175 nodes (proteins) and 285 edges (interactions). Functional enrichment analysis showed that the proteins in this netwirk are significantly enriched for the GO term 'negative regulation of cell differentiation' (FDR<0.05). This category included proteins such as noggin (NOG), LIM domain only 2 (LMO2), v-maf avian musculoaponeurotic fibrosarcoma oncogene homolog B (MAFB), delta-like 3 (DLL3), tumor protein p63 (TP63), Toll-like receptor 3 (TLR3), nuclear receptor subfamily 2, group E, member 1 (NR2E1), parathyroid hormone-like hormone (PTHLH), brain-derived neurotrophic factor (BDNF), PR domain containing 6 (PRDM6), inositol polyphosphate-5-phosphatase(INPP5D), chordin (CHRD), and twist basic helix-loop-helix transcription factor 2 (TWIST2).

PPI network. Fig. 4 shows the PPI network of DEGs in metastatic GC, which contained 191 nodes and 474 edges. The number of downregulated proteins was higher than that of upregulated proteins. Moreover, downregulated proteins were often observed as components of the same module, such as in module 1 in Fig. 5. A total of 6 modules were identified from the entire network (Fig. 5) using ClusterONE. Module 1 comprised 32 downregulated genes products and 245 interactions. Functional enrichment analysis showed that proteins of this module were significantly enriched for immune functions, such as immune response $(\mathrm{FDR}<0.05)$ and antigen processing and presentation of peptide antigen via MHC class I $($ FDR $<0.05)$. No significant enrichment for GO terms was observed in the remaining 5 modules. However, STAT1 

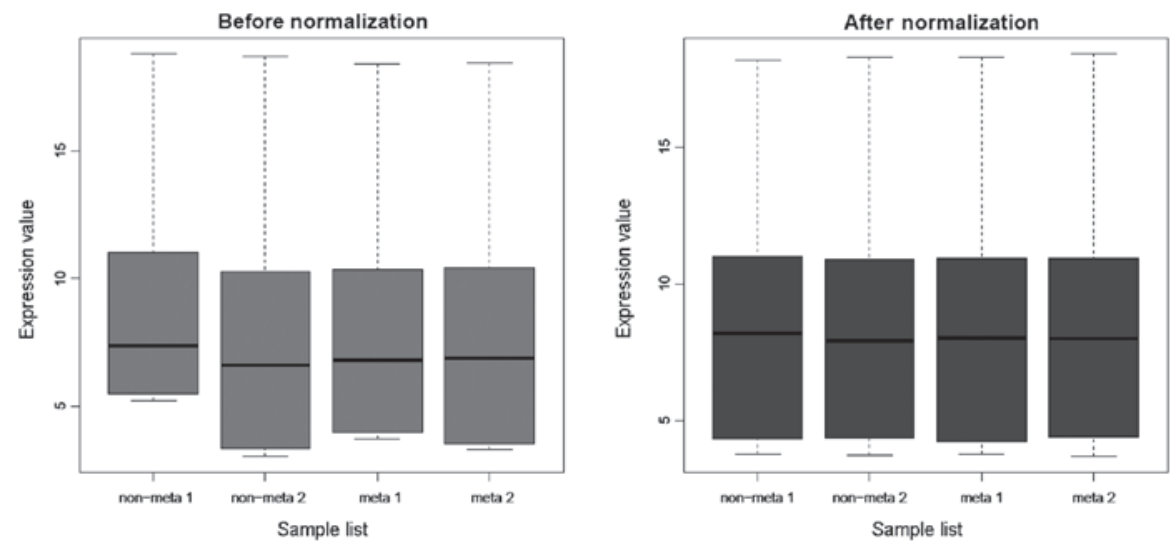

Figure 1. Boxplots of gene expression data before (left) and after normalization (right). The medians (horizontal black lines) are nearly at the same level, indicating a good performance of normalization. Meta, metastasis.

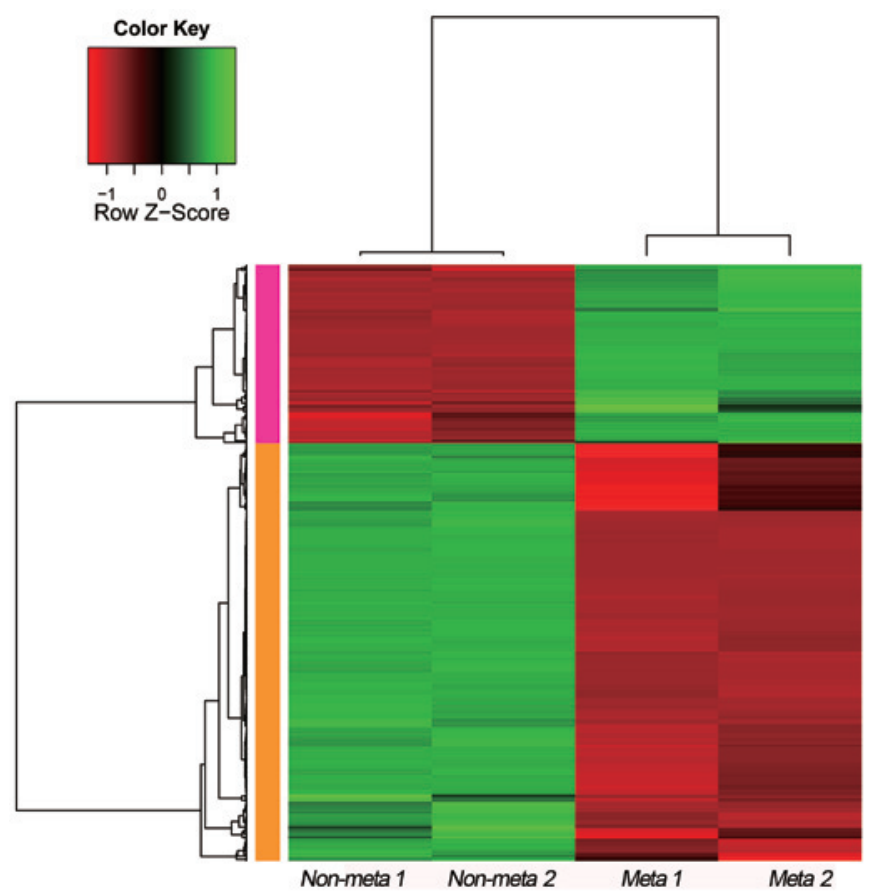

Figure 2. Clustering analysis of differentially expressed genes. Data were derived from the $\log _{2}$ transformation for each gene. Red, high expression; green, low expression, according to $\mathrm{z}$ scores. Meta, metastasis.

Table I. GO enrichment analysis results for upregulated and downregulated genes.

\begin{tabular}{llccc}
\hline Category & \multicolumn{1}{c}{ GO term } & Count & P-value & FDR \\
\hline Upregulated genes & 0006805-xenobiotic metabolic process & 5 & $3.88 \mathrm{E}-02$ & $4.64 \mathrm{E}-02$ \\
Downregulated genes & 0006955-immune response & 45 & $8.13 \mathrm{E}-09$ & $6.86 \mathrm{E}-09$ \\
& 0009615-response to virus & 16 & $7.17 \mathrm{E}-06$ & $6.05 \mathrm{E}-06$ \\
& 0019882-antigen processing and presentation & 13 & $1.56 \mathrm{E}-04$ & $1.31 \mathrm{E}-04$ \\
& 0000904-cell morphogenesis involved in differentiation & 17 & $5.50 \mathrm{E}-02$ & $4.77 \mathrm{E}-02$ \\
& 0005615-extracellular space & 35 & $3.91 \mathrm{E}-04$ & $1.96 \mathrm{E}-03$ \\
& 0044421-extracellular region part & 42 & $1.38 \mathrm{E}-03$ & $6.91 \mathrm{E}-03$ \\
& 0005576-extracellular region & 69 & $3.08 \mathrm{E}-03$ & $1.55 \mathrm{E}-02$ \\
& 0042611-MHC protein complex & 9 & $5.05 \mathrm{E}-03$ & $2.54 \mathrm{E}-02$ \\
& 0009986-cell surface & 21 & $8.53 \mathrm{E}-03$ & $4.29 \mathrm{E}-02$
\end{tabular}

Count, the number of differentially expressed genes. FDR, false discovery rate; GO, Gene Ontology. 


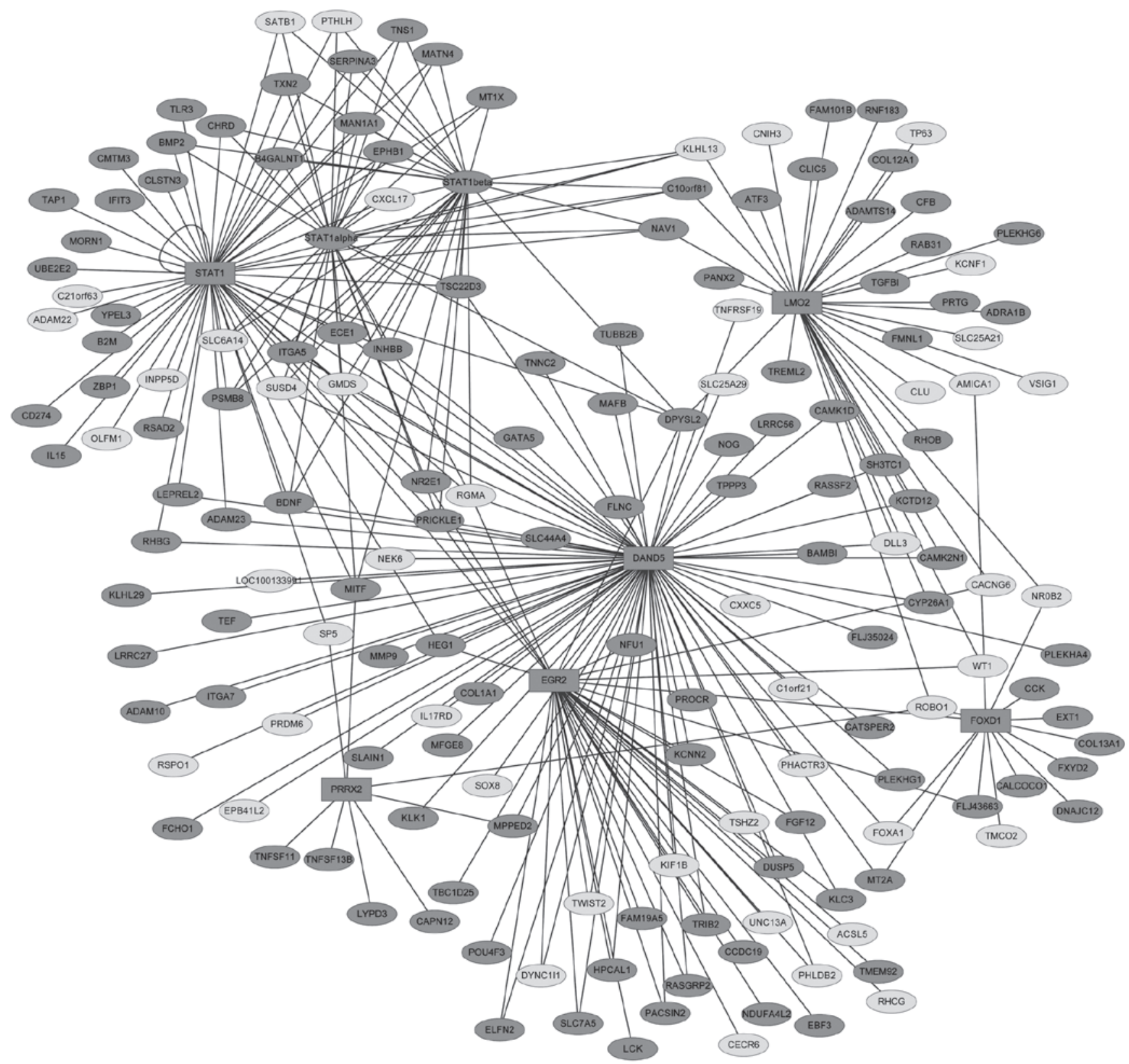

Figure 3. The transcriptional regulatory network of differentially expressed genes in metastatic gastric cancer. Dark rectangles represent transcription factors $(n=6)$, gray ellipses upregulated target genes $(n=49)$, and dark ellipses downregulated target genes $(n=120)$.

was found to be part of two modules (1 and 2), suggesting that this protein may have a role in the development of metastatic GC.

\section{Discussion}

In the present study, a total of 584 DEGs were identified in metastatic GC, of which 175 were upregulated and 409 downregulated. GO enrichment analysis revealed that the downregulated genes were significantly enriched for immune response and relevant pathways. According to the transcriptional regulatory network analysis, 6 DEGs were found to be TFs, associated with 169 predicted targets. Functional enrichment analysis revealed that the genes in this network are enriched for negative regulation of cell differentiation. In addition, 6 functional modules were extracted from the PPI network; of these modules, module 1 showed a significant overrepresentation for immune response and relevant biological functions.

Immune response is closely related to cancer, while immune escape is a critical gateway to malignancy (21). The study by Maehara et al (22) confirmed that there is a negative correlation between lymph node metastasis and dendritic cell infiltration in GC. In agreement with previous studies, a number of downregulated genes in metastatic GC were found to be involved in immune response, as well as antigen processing and presentation of peptide antigen via major histocompatibility complex (MHC) class I (Fig. 5, module 1). Downregulation of human leukocyte antigen (HLA) class I antigen processing molecules is considered to relate to renal cell carcinoma (23). Cabrera et al (24) found that HLA class I expression in metastatic melanoma correlates to tumor development during 


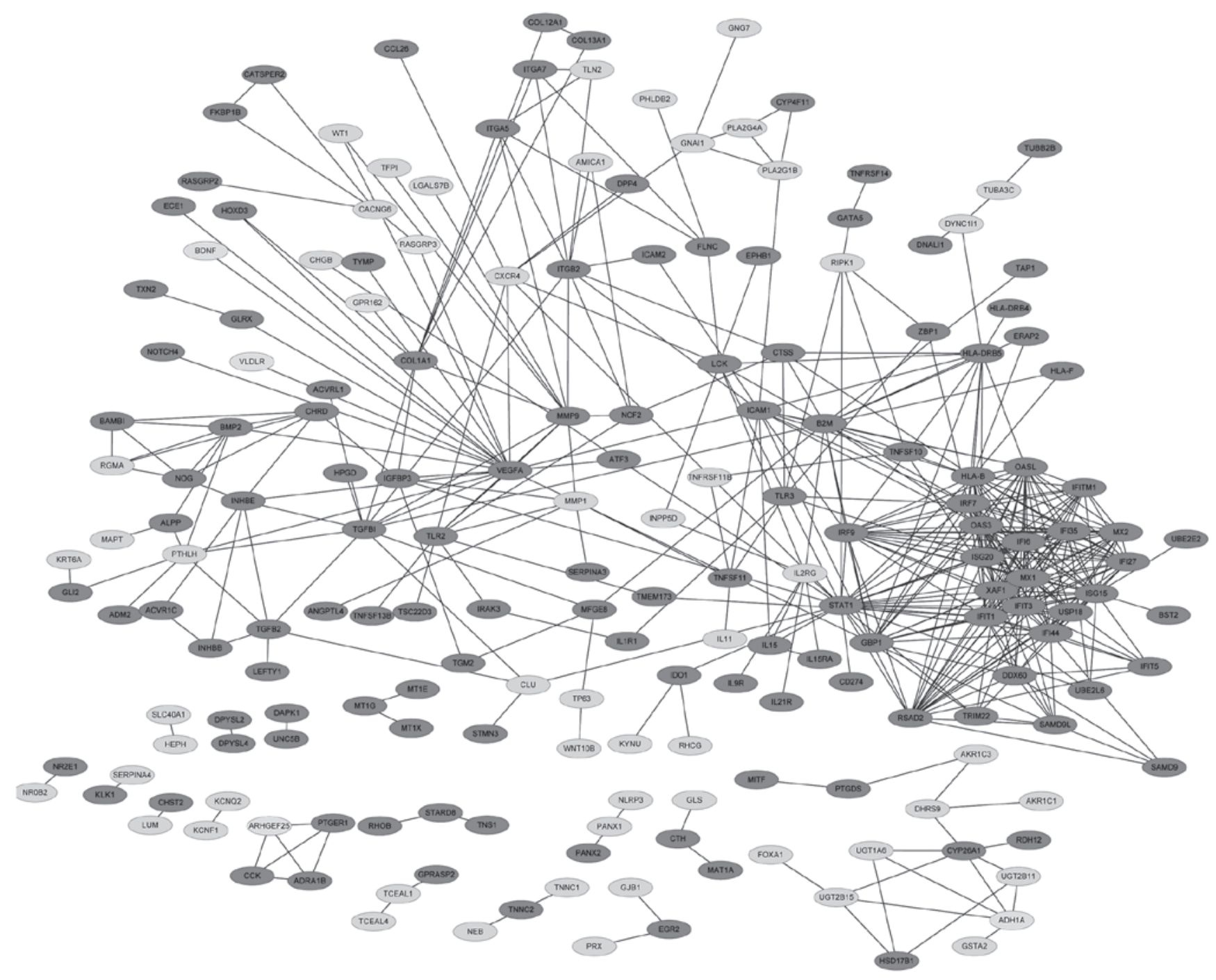

Figure 4. The protein-protein interaction network of differentially expressed gene products in metastatic gastric cancer. Downregulated proteins in metastatic $\mathrm{GC}$ are in dark $(\mathrm{n}=133)$, upregulated proteins in gray $(\mathrm{n}=55)$, and non-differentially expressed ones in light gray $(\mathrm{n}=6)$.

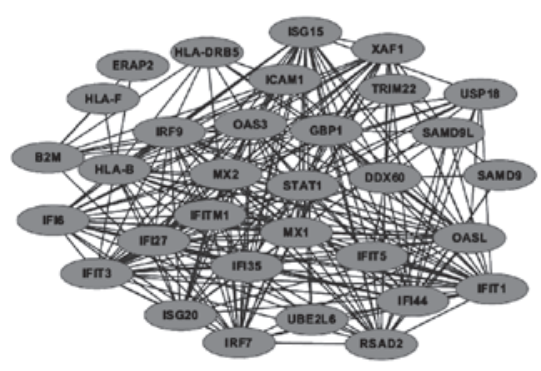

module 1

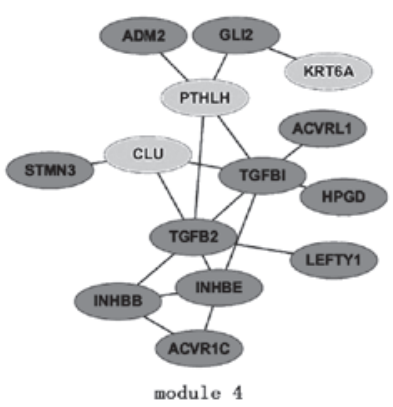

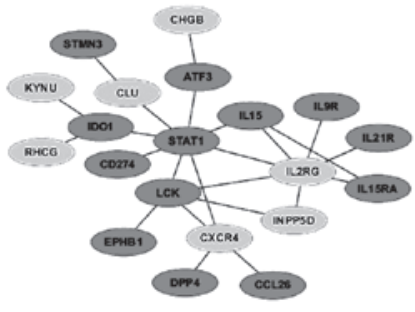

module 2

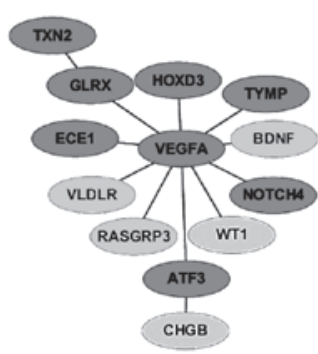

module 5
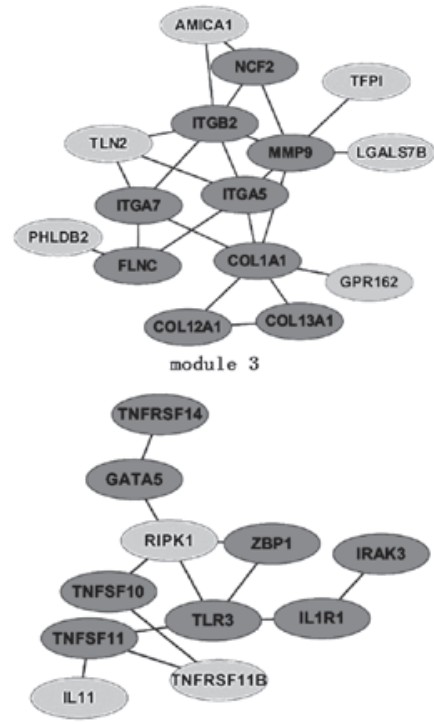

module 6

Figure 5. Six functional modules identified from the entire protein-protein interaction network using ClusterONE. Downregulated proteins in metastatic gastric cancer are in dark $(n=81)$, upregulated proteins in gray $(n=20)$, and non-differentially expressed ones in light gray $(n=4)$. 
autologous vaccination. A decrease in the expression of class I proteins was also reported in metastases of colorectal, gastric and laryngeal carcinomas (25). In the present study, several members of the HLA family were identified as downregulated, such as MHC class I, J (HLA-J), MHC class I, B (HLA-B) and MHC class I, F (HLA-F). Downregulation of HLA-B was also observed in metastatic serous adenocarcinomas, although the difference was not statistically significant (26).

Dedifferentiation is related to metastasis $(27,28)$. Certain of the proteins encoded by the DEGs that are associated with this term have been reported to be linked to metastasis. Nakata et al (29) showed that LMO2 expression is related to aggressive behavior and distant metastasis in prostate cancer. TP63 is a suppressor of tumorigenesis and metastasis interacting with the mutant p53 protein (30). TLR3 is a member of transmembrane proteins that recognize conserved molecular motifs of viral and bacterial origin and initiate the innate immune response. Zhang et al (31) reported that TLR3 activation inhibits nasopharyngeal carcinoma metastasis via downregulation of the chemokine receptor CXCR4. González-Reyes et al (32) asserted that the expression levels of TLR3, TLR4 and TLR9 have clinical value as indicators of tumor aggressiveness in breast cancer. TWIST2 is implicated in cell lineage determination and differentiation. Fang et al (33) reported that TWIST2 contributes to breast cancer progression by promoting the epithelial-mesenchymal transition and cancer stem-like cell self-renewal. Similar results were observed in cervical carcinoma (34). Future studies on the proteins encoded by these DEGs may provide interesting findings and data for the development of new therapeutic targets for metastatic GC.

Six TFs were identified from the DEGs and these could regulate 169 target genes, forming 175 nodes in the transcriptional regulatory network (Fig. 3). Previous studies have shed light on their roles in the development of metastatic cancers. Ernst et al (35) found that STAT3 and STAT1 mediate IL-11dependent and inflammation-associated gastric tumorigenesis in gp130 receptor-mutant mice. In addition, STAT1 is also related to metastasis. Khodarev et al (36) point out that the STAT1 pathway mediates amplification of the metastatic potential and resistance to therapy. In cultured cell-based experiments, Greenwood et al (37) reported that activation of STAT1 causes increased migration and invasion, and increases the abundance of CD74. CD74 overexpression leads to increased membrane expression of proteins involved in cell adhesion and metastasis. The protein inhibitor of activated STAT1 (PIAS1) is a novel modulator of the JAK/STAT signaling pathway that negatively regulates the inflammatory response. The study by Chen et al (38) showed that PIAS1 is downregulated in GC tissueS and involved in cell metastasis. However, according to the study by Huang et al (39), STAT1 is a negative regulator of tumor angiogenesis and, hence, of tumor growth and metastasis. STAT1 is an important TF regulating a range of target genes. Diverse signaling pathways dominate the physiological processes at different cellular conditions, which may explain the conflicting roles reported for STAT1 in cancer. Research on deregulated target genes of STAT1 may enhance the current understanding on the regulatory mechanisms this TF is involved in. Certain of the target genes of STAT1 have been previously associated with cancer metastasis. The gene encoding the bone morphogenetic protein-2 (BMP-2) is one of these: Park et al (40) reported that BMP-2 is associated with progression to a metastatic state in GC. The study by Kang et al (41) further pointed out that the BMP-2 signaling pathway enhances tumor metastasis by sequential activation of the PI3K/AKT or the MAPK pathways, followed by the induction of nuclear factor- $\kappa \mathrm{B}$ and MMP-9. The chemokine C-X-C motif ligand 17 gene (CXCL17) is another transcriptional target of STAT1. CXCL17 was found to be upregulated in metastatic GC in our data. Matsui et al (42) indicated that the CXCL17 protein recruits immature myeloidderived cells in tumor cells, and promotes tumor progression through angiogenesis. Future research on these target genes may reveal new therapeutic targets for metastatic GC.

EGR2 is a transcription factor with three tandem C2H2-type zinc fingers. It has been identified as a tumor suppressor, and its expression level is decreased in various types of cancer $(43,44)$. miR-150 promotes GC proliferation by negatively regulating the pro-apoptotic gene EGR2 (45). LaTulippe et al (46) reported that $E G R 2$ and $E G R 3$ are differentially expressed at least 3-fold between primary and metastatic prostate cancer. ERG2 has numerous target genes, among which TWIST2, which was previously linked to metastasis (47). The RGM domain family member A (RGMA), a member of the repulsive guidance molecule family, is also a target gene of ERG2. RGMA is regarded as a key regulator of growth and aggressiveness of prostate cancer cells (48). Zhao et al (49) showed that decreased expression of $R G M A$ by DNA methylation in colorectal cancer is related to tumor progression. In our data, $R G M A$ was downregulated in metastatic GC. We therefore hypothesize that this gene may play a role in metastasis of GC cells.

Overall, the present study described the differential expression profiles between metastatic and non-metastatic GC. The results of this study provided meaningful data for future investigations; the identified DEGs will be useful in future research aiming to elucidate the regulatory mechanism underlying metastasis. Among these DEGs, STAT1 and EGR2 are promising candidates for use as metastatic GC biomarkers, and thus warrant further investigation.

\section{Acknowledgements}

This study was supported by a grant from the Natural Scientific Foundation of Shanghai (no. 13ZR1413500). We wish to express our warm acknowledgement to Lishan Wang from the Shanghai Jiao Tong University. His ideas and help added a valuable dimension to this study.

\section{References}

1. World Health Organization: Cancer. Fact sheet 297. WHO, Geneva, Switzerland 2011.

2. Yonemura Y, Endo Y, Fujita H, et al: Role of vascular endothelial growth factor $\mathrm{C}$ expression in the development of lymph node metastasis in gastric cancer. Clin Cancer Res 5: 1823-1829, 1999.

3. Shimizu K, Kubo H, Yamaguchi K, et al: Suppression of VEGFR-3 signaling inhibits lymph node metastasis in gastric cancer. Cancer Sci 95: 328-333, 2004.

4. Xu X, Abuduhadeer X, Zhang WB, et al: Knockdown of RAGE inhibits growth and invasion of gastric cancer cells. Eur J Histochem 57: e36, 2013.

5. Malafa M, Margenthaler J, Webb B, Neitzel L and Christophersen M: MnSOD expression is increased in metastatic gastric cancer. J Surg Res 88:130-134, 2000. 
6. Tang B, Peng ZH, Yu PW, Yu G and Qian F: Expression and significance of $\mathrm{Cx} 43$ and E-cadherin in gastric cancer and metastatic lymph nodes. Med Oncol 28: 502-508, 2011.

7. Hippo Y, Taniguchi H, Tsutsumi S, et al: Global gene expression analysis of gastric cancer by oligonucleotide microarrays. Cancer Res 62: 233-240, 2002.

8. Kang HC, Kim IJ, Park JH, et al: Identification of genes with differential expression in acquired drug-resistant gastric cancer cells using high-density oligonucleotide microarrays. Clin Cancer Res 10: 272-284, 2004.

9. Yamashita S, Tsujino Y, Moriguchi K, Tatematsu M and Ushijima T: Chemical genomic screening for methylation-silenced genes in gastric cancer cell lines using 5-aza-2'-deoxycytidine treatment and oligonucleotide microarray. Cancer Sci 97: 64-71, 2006.

10. Edgar R, Domrachev M and Lash AE: Gene Expression Omnibus: NCBI gene expression and hybridization array data repository. Nucleic Acids Res 30: 207-210, 2002.

11. Hatakeyama K, Ohshima K, Fukuda Y, et al: Identification of a novel protein isoform derived from cancer-related splicing variants using combined analysis of transcriptome and proteome. Proteomics: 11: 2275-2282, 2011.

12. Gautier L, Cope L, Bolstad BM and Irizarry RA: affy - analysis of Affymetrix GeneChip data at the probe level. Bioinformatics 20 307-315, 2004

13. Smyth GK: Limma: linear models for microarray data. In: Bioinformatics and Computational Biology Solutions Using R and Bioconductor. Springer, New York, pp397-420, 2005.

14. Eisen MB, Spellman PT, Brown PO, Botstein D: Cluster analysis and display of genome-wide expression patterns. Proc Natl Acad Sci USA 95:14863-14868, 1998.

15. Page RD: TreeView: an application to display phylogenetic trees on personal computers. Comput Appl Biosci 12: 357-358, 1996.

16. Huang da W, Sherman BT, Tan Q, et al: The DAVID Gene Functional Classification Tool: a novel biological module-centric algorithm to functionally analyze large gene lists. Genome Biol 8: R183, 2007.

17. Chan PP, Holmes AD, Smith AM, Tran D and Lowe TM: The UCSC Archaeal Genome Browser: 2012 update. Nucleic Acids Res 40: D646-D652, 2012.

18. Kanehisa M and Goto S: KEGG: kyoto encyclopedia of genes and genomes. Nucleic Acids Res: 28: 27-30, 2000.

19. Shannon P, Markiel A, Ozier O, et al: Cytoscape: a software environment for integrated models of biomolecular interaction networks. Genome Res 13: 2498-2504, 2003.

20. Nepusz T, Yu H and Paccanaro A: Detecting overlapping protein complexes in protein-protein interaction networks. Nat Methods 9:471-472, 2012.

21. Prendergast G: Immune escape as a fundamental trait of cancer: focus on IDO. Oncogene 27: 3889-3900, 2008.

22. Maehara Y, Tomisaki S, Oda S, et al: Lymph node metastasis and relation to tumour growth potential and local immune response in advanced gastric cancer. Int J Cancer 74: 224-228, 1997.

23. Atkins D, Ferrone S, Schmahl GE, Störkel S and Seliger B: Down-regulation of HLA class I antigen processing molecules: an immune escape mechanism of renal cell carcinoma? J Urol 171: 885-889, 2004.

24. Cabrera T, Lara E, Romero JM, et al: HLA class I expression in metastatic melanoma correlates with tumor development during autologous vaccination. Cancer Immunol Immunother 56 709-717, 2007.

25. López-Nevot MA, Esteban F, Ferrón A, Gutiérrez J, et al: HLA class I gene expression on human primary tumours and autologous metastases: demonstration of selective losses of HLA antigens on colorectal, gastric and laryngeal carcinomas. $\mathrm{Br}$ J Cancer 59: 221-226, 1989.

26. Lee YS, Kim TE, Kim BK, et al: Alterations of HLA class I and class II antigen expressions in borderline, invasive and metastatic ovarian cancers. Exp Mol Med 34: 18-26, 2002.

27. Grünert S, Jechlinger $M$ and Beug H: Diverse cellular and molecular mechanisms contribute to epithelial plasticity and metastasis. Nat Rev Mol Cell Biol 4: 657-665, 2003.

28. Kaihara T, Kusaka T, Nishi M, et al: Dedifferentiation and decreased expression of adhesion molecules, E-cadherin and $\mathrm{ZO}-1$, in colorectal cancer are closely related to liver metastasis. J Exp Clin Cancer Res 22: 117-123, 2003.
29. Nakata K, Ohuchida K, Nagai E, et al: LMO2 is a novel predictive marker for a better prognosis in pancreatic cancer. Neoplasia 11: 712-719, 2009.

30. Melino G: p63 is a suppressor of tumorigenesis and metastasis interacting with mutant p53. Cell Death Differ 18: 1487-1499, 2011.

31. Zhang Y, Sun R, Liu B, et al: TLR3 activation inhibits nasopharyngeal carcinoma metastasis via down-regulation of chemokine receptor CXCR4. Cancer Biol Ther 8: 1826-1830, 2009.

32. González-Reyes S, Marín L, González L, et al: Study of TLR3, TLR4 and TLR9 in breast carcinomas and their association with metastasis. BMC Cancer 10: 665, 2010.

33. Fang X, Cai Y, Liu J, et al: Twist2 contributes to breast cancer progression by promoting an epithelial-mesenchymal transition and cancer stem-like cell self-renewal. Oncogene 30: 4707-4720, 2011.

34. Li Y, Wang W, Wang W, et al: Correlation of TWIST2 up-regulation and epithelial-mesenchymal transition during tumorigenesis and progression of cervical carcinoma. Gynecol Oncol 124: 112-118, 2012.

35. Ernst M, Najdovska M, Grail D, et al: STAT3 and STAT1 mediate IL-11-dependent and inflammation-associated gastric tumorigenesis in gp130 receptor mutant mice. J Clin Invest 118: $1727-1738,2008$

36. Khodarev NN, Roach P, Pitroda SP, et al: STAT1 pathway mediates amplification of metastatic potential and resistance to therapy. PLoS One 4: e5821, 2009.

37. Greenwood C, Metodieva G, Al-Janabi K, et al: Stat1 and CD74 overexpression is co-dependent and linked to increased invasion and lymph node metastasis in triple-negative breast cancer. J Proteomics 75: 3031-3040, 2012

38. Chen P, Zhao D, Sun Y, Huang L, Zhang S and Yuan Y: Protein inhibitor of activated STAT-1 is downregulated in gastric cancer tissue and involved in cell metastasis. Oncol Rep 28: 2149-2155, 2012.

39. Huang S, Bucana CD, Van Arsdall M and Fidler IJ: Stat1 negatively regulates angiogenesis, tumorigenicity and metastasis of tumor cells. Oncogene 21: 2504-2512, 2002.

40. Park Y, Kim JW, Kim DS, et al: The bone morphogenesis protein-2 (BMP-2) is associated with progression to metastatic disease in gastric cancer. Cancer Res Treat 40: 127-132, 2008.

41. Kang MH, Oh SC, Lee HJ, et al: Metastatic function of BMP-2 in gastric cancer cells: the role of PI3K/AKT, MAPK, the NF- $\mathrm{BB}$ pathway, and MMP-9 expression. Exp Cell Res 317: 1746-1762, 2011.

42. Matsui A, Yokoo H, Negishi Y, et al: CXCL17 expression by tumor cells recruits $\mathrm{CD} 11 \mathrm{~b}^{+} \mathrm{Gr} 1^{\text {high }} \mathrm{F} 4 / 80^{-}$cells and promotes tumor progression. PLoS One 7: e44080, 2012.

43. Unoki $M$ and Nakamura Y: EGR2 induces apoptosis in various cancer cell lines by direct transactivation of BNIP3L and BAK. Oncogene 22: 2172-2185, 2003.

44. Nakahara Y, Shiraishi T, Okamoto H, et al: Detrended fluctuation analysis of genome-wide copy number profiles of glioblastomas using array-based comparative genomic hybridization. Neuro Oncol 6: 281-289, 2004

45. Wu Q, Jin H, Yang Z, et al: MiR-150 promotes gastric cancer proliferation by negatively regulating the pro-apoptotic gene EGR2. Biochem Biophys Res Commun 392: 340-345, 2010.

46. LaTulippe E, Satagopan J, Smith A, et al: Comprehensive gene expression analysis of prostate cancer reveals distinct transcriptional programs associated with metastatic disease. Cancer Res 62: 4499-4506, 2002.

47. Li Y, Wang W, Wang W, et al: Correlation of TWIST2 up-regulation and epithelial-mesenchymal transition during tumorigenesis and progression of cervical carcinoma. Gynecol Oncol 124: 112-118, 2012.

48. Li J, Ye L, Kynaston HG and Jiang WG: Repulsive guidance molecules, novel bone morphogenetic protein co-receptors, are key regulators of the growth and aggressiveness of prostate cancer cells. Int J Oncol 40: 544-550, 2012

49. Zhao ZW, Lian WJ, Chen GQ, et al: Decreased expression of repulsive guidance molecule member A by DNA methylation in colorectal cancer is related to tumor progression. Oncol Rep 27: $1653-1659,2012$ 